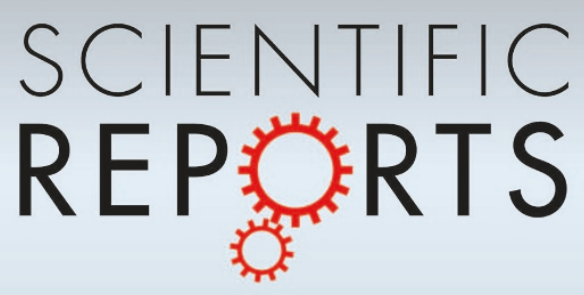

OPEN

SUBJECT AREAS:

CHARACTERIZATION AND ANALYTICAL

TECHNIQUES

SUB-WAVELENGTH OPTICS

SURFACE PATTERNING

NANOPHOTONICS AND

PLASMONICS

Received

12 March 2014

Accepted

16 June 2014

Published

2 July 2014

Correspondence and requests for materials should be addressed to M.B. (michel.bosman@ gmail.com) or

J.K.W.Y. (joel_yang@ sutd.edu.sg)

* These authors contributed equally to this work.

\section{Encapsulated Annealing: Enhancing the Plasmon Quality Factor in Lithographically-Defined Nanostructures}

\author{
Michel Bosman ${ }^{1 *}$, Lei Zhang ${ }^{2 *}$, Huigao Duan ${ }^{3}$, Shu Fen Tan ${ }^{4}$, Christian A. Nijhuis ${ }^{1,4,5,6}$, Cheng-Wei Qiu ${ }^{2}$ \\ \& Joel K. W. Yang ${ }^{1,7}$
}

\begin{abstract}
'Institute of Materials Research and Engineering, A*STAR (Agency for Science, Technology and Research), 3 Research Link, Singapore 1 17602, ${ }^{2}$ Department of Electrical and Computer Engineering, National University of Singapore, 4 Engineering Drive 3, Singapore $117583,{ }^{3}$ College of Physics and Microelectronics, State Key Laboratory of Chemo/Biosensing and Chemometrics, Hunan University, Changsha 410082, China, ${ }^{4}$ Department of Chemistry, National University of Singapore, 3 Science Drive 3, Singapore 117543,5 Solar Energy Research Institute of Singapore (SERIS), 7 Engineering Drive 1, National University of Singapore, Singapore 117574, Singapore, ${ }^{6}$ Graphene Research Centre, National University of Singapore, 2 Science Drive 3, Singapore $117542,{ }^{7}$ Singapore University of Technology and Design (SUTD), 20 Dover Drive, Singapore 138682.
\end{abstract}

Lithography provides the precision to pattern large arrays of metallic nanostructures with varying geometries, enabling systematic studies and discoveries of new phenomena in plasmonics. However, surface plasmon resonances experience more damping in lithographically-defined structures than in chemicallysynthesized nanoparticles of comparable geometries. Grain boundaries, surface roughness, substrate effects, and adhesion layers have been reported as causes of plasmon damping, but it is difficult to isolate these effects. Using monochromated electron energy-loss spectroscopy (EELS) and numerical analysis, we demonstrate an experimental technique that allows the study of these effects individually, to significantly reduce the plasmon damping in lithographically-defined structures. We introduce a method of encapsulated annealing that preserves the shape of polycrystalline gold nanostructures, while their grain-boundary density is reduced. We demonstrate enhanced Q-factors in lithographically-defined nanostructures, with intrinsic damping that matches the theoretical Drude damping limit.

1 hy do plasmonic structures have poorer performance when they are fabricated with top-down techniques compared to those fabricated with bottom-up approaches?

Surface plasmon resonances produce strong electric fields within sub-diffraction-limited volumes that can be applied in various nanophotonic devices. Using top-down lithographic approaches such as electron-beam lithography (EBL), these resonators can be patterned deterministically over large arrays with sub-10 nanometer dimensional control ${ }^{1-3}$. As a universal damping parameter for resonators, the quality-factor (Q-factor) has been extensively studied with different plasmonic nanostructures ${ }^{4,5}$. Unfortunately, in addition to damping due to the intrinsic properties of the material, extrinsic sources such as grain boundaries, surface roughness, and adhesion layers $^{6-10}$ also introduce damping and suppress Q-factors in lithographically-defined nanostructures. In contrast, wet-chemically-synthesized metal nanoparticles can be monocrystalline and atomically smooth ${ }^{11,12}$ and possess higher Q-factors. However, due to the challenges in precise placement of these particles, lithography provides an acceptable compromise for plasmonic device applications. To enhance Q-factors of lithographically-defined structures, thermal annealing ${ }^{7,13,14}$, and substituting metal adhesion layers with organic ligands ${ }^{9,15}$ have shown promise. However, more detailed studies are needed, e.g. to anneal-out grain boundaries without shape change, and suppress Rayleigh instabilities that break up long and narrow nanostructures ${ }^{16,17}$. As high Q-factor plasmonic resonators are useful in practical applications such as surface-enhanced Raman scattering (SERS) ${ }^{18}$, color printing $^{19}$, and light extraction from quantum emitters ${ }^{20}$, efforts to improve Q-factors are relevant.

In this work, we present a readily-applicable method $^{21}$ that combines the design freedom of top-down lithography and the high Q-factors of chemically-synthesized nanoparticles, where the intrinsic damping is at 
the Drude limit ${ }^{7,11,22}$. By incorporating a thermally-stable but easily removable encapsulation layer, the EBL-defined nanostructures can be annealed to reduce grain boundaries without changing the geometry or surface roughness of the nanostructures. We applied this method to isolate and analyze the contributions of the various damping sources in plasmonic nanostructures. Ensemble-averaging effects were avoided in our analysis by studying single nanostructures with monochromated electron energy-loss spectroscopy (EELS) in a transmission electron microscopy (TEM) ${ }^{23-28}$. Interestingly, our results show that despite the incomplete removal of grain boundaries, annealed nanostructures have damping parameters close to those of monocrystalline nanoparticles, matching the Drude damping parameter of bulk metal. We also show the importance of a hitherto unexamined parameter, i.e. the contact area between the nanostructure and a dielectric substrate.

\section{Results}

Figure 1 shows the process flow for reducing the grain boundary density of nanostructures. Au nanostructures with a thickness of $25 \mathrm{~nm}$ were fabricated directly onto $30 \mathrm{~nm}$ thick, free-standing $\mathrm{Si}_{3} \mathrm{~N}_{4}$ TEM support membranes ${ }^{29,30}$. A $30 \mathrm{~nm}$ thick layer of hydrogen silsesquioxane (HSQ, XR-1541 2\% solids) was then spin-coated onto the samples. Thermal annealing was performed in a Jipelek rapid thermal processor under nitrogen environment at $400^{\circ} \mathrm{C}$ for 10 minutes. After annealing, the HSQ encapsulation was removed by immersing the sample in a $2 \%$ vol. solution of hydrofluoric acid (HF) for $15 \mathrm{~s}$. The shape and surface roughness of the nanostructures were preserved due to the encapsulation. In contrast, control samples without encapsulation experienced changes in shape, size, and surface roughness ${ }^{16}$ as can be seen in Fig. S1 of the Supplementary Information (SI).

The nanostructures were fabricated onto $\mathrm{Si}_{3} \mathrm{~N}_{4}$ membranes to enable the direct high-resolution imaging of the grain boundaries. Figure 2 shows TEM images of ring-and rod-shaped nanostructures with a $1 \mathrm{~nm} \mathrm{Cr}$ adhesion layer, before and after annealing. The highly-polycrystalline nature of the gold nanostructures prior to annealing is seen in the TEM image of Fig. 2(a) with grain sizes of $\sim 10 \mathrm{~nm}$. Grain boundaries are marked by dashed, yellow lines in the close-up panels. As shown in Fig. 2(b), the same region after annealing had fewer grain boundaries, likely due to grain-boundary migration and Ostwald ripening at elevated temperatures ${ }^{31}$. The average

(a)

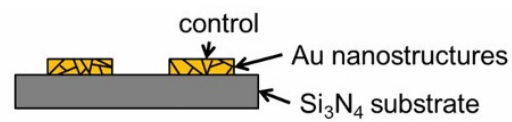

(b) encapsulate with hydrogen silsesquioxane (HSQ)

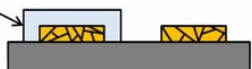

(c) thermal anneal

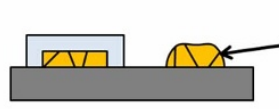

control sample undergoes

(c) thermal anneal

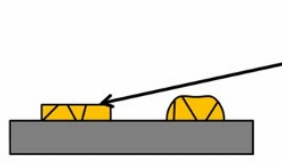
undesired shape change

(d) remove $\mathrm{HSQ}$

preserved shape but reduced grain boundaries

Figure $1 \mid$ Process flow for reducing grain boundaries of lithographically-defined nanostructures with minimal change in their shape and surface roughness. (a) Gold nanostructures on $30 \mathrm{~nm}$ thick, amorphous $\mathrm{Si}_{3} \mathrm{~N}_{4}$ membranes. (b) Encapsulation by a $30 \mathrm{~nm}$ thick layer of HSQ. (c) Annealing at $400^{\circ} \mathrm{C}$ for 10 minutes. (d) Immersing the sample in dilute HF to remove the encapsulating HSQ. diameter of the grains increased to $\sim 20 \mathrm{~nm}$, leading to a significant reduction of the total number of crystals, as evidenced by the reduced number of bright spots in the electron diffraction patterns (bottom panels, Fig. 2(a) and (b)).

Shape preservation was afforded by HSQ encapsulation and not by adhesion layer pinning. An example of a structure fabricated without $\mathrm{Cr}$ is shown in Fig. 2(c). The length of this nanorod was $454 \mathrm{~nm}$ before and $446 \mathrm{~nm}$ after annealing, a reduction in length of $\sim 2 \%$. The edge profiles of the nanorod were still preserved by the encapsulation, confirming the minimal effect of the process on the shape and surface roughness of the nanostructures.

EELS measurements were done on the same structures both before and after they were annealed; the experimental procedures are described in the SI. EELS results for the nanorod of Fig. 2(c) are presented in Fig. 3(a). Up to four peaks corresponding to different resonant harmonic modes are evident, and their corresponding plasmon maps are shown beneath the respective peaks. The effects of annealing are evident in the enhanced intensity and narrowed fullwidth at half maximum (FWHM) of the resonant peaks. Q-factors were extracted from the spectra by using a previously-described method $^{32}$. The blue shift of the plasmon peaks observed in Fig. 3(a) after annealing is likely caused by a combination of factors, including the reduction of the rod length during annealing, and possibly the thinning of the substrate during etching, as is shown in more detail in Fig. S3 in the SI.

Numerical calculations using FDTD were performed to extract the Q-factors and field distributions of the nanostructures, as described in the Methods section. The relative permittivity for bulk Au was described using the Drude-Lorentz model ${ }^{7}$, expressed as follows:

$$
\varepsilon=1-\frac{\omega_{p}^{2}}{\omega^{2}+i \alpha \Gamma_{p} \omega}+\sum_{m} \frac{f_{m} \omega_{m}^{2}}{\omega_{m}^{2}-\omega^{2}-i \Gamma_{m} \omega}
$$

Here $\omega_{p}$ is the plasma frequency, $\Gamma_{p}$ is the damping parameter for the Drude term, $\omega_{m}$, and $\Gamma_{m}$ are the resonant frequency and the damping parameter of the $m^{\text {th }}$ Lorentz oscillator, respectively. The increase in damping due to factors such as grain boundaries and impurities is accounted for by an increase in the Drude damping parameter. Following the work of Liu et al. ${ }^{10}$, a damping factor $\alpha$ was introduced to quantify this increase relative to bulk Au, where $\alpha=1$ is defined to be at the Drude limit. By fitting experimental permittivity data ${ }^{33}$ with two Lorentzian terms, we obtained the following parameters: $\omega_{p}=9 \mathrm{eV}, \alpha=1, \Gamma_{p}=0.07 \mathrm{eV}, f_{1}=$ $0.3, \omega_{1}=2.7 \mathrm{eV}, \Gamma_{1}=0.3 \mathrm{eV}, f_{2}=0.8, \omega_{2}=3.05 \mathrm{eV}$ and $\Gamma_{2}$ $=0.5 \mathrm{eV}$, as shown in Fig. S2 in the SI. Figure 3(b) shows spectra for the fundamental mode of a $100 \mathrm{~nm}$ long gold rod with $\alpha=1$, 1.3, and 1.8. Q-factors were extracted from fitted Lorentzian functions to the simulated spectra ${ }^{4}$.

Experimental Q-factors extracted from three sets of gold nanostructures are shown in Fig. 3(c) as a function of resonance energy. To verify the effects of the adhesion layer and grain boundaries on the damping process, we compared these experimental results with simulated Q-factors of nanostructures with lengths from 40 to $275 \mathrm{~nm}$ and square cross sections $25 \mathrm{~nm}$ wide. Structures with $\mathrm{Cr}$ adhesion have the lowest Q-factors, and could be fitted with a damping factor of $\alpha=1.8$. Without $\mathrm{Cr}, \mathrm{Q}$-factors immediately improved by $\sim 25 \%$. Notably, samples with $\mathrm{Cr}$ adhesion did not show a significant increase in Q-factor $(<10 \%)$ after annealing, though it was evident that they experienced a reduction in grain boundary densities, as seen in Fig. 2. It is likely that $\mathrm{Cr}$ diffuses into the gold during annealing, to form $\mathrm{Cr}$-rich crystallites ${ }^{34}$, creating new scattering sites that compensate the reduction in damping from grain boundaries. Conversely, the samples without Cr exhibited an improvement in Qfactor by as much as $\sim 40 \%$ after annealing (Fig. 3(c) inset). The asfabricated structures without $\mathrm{Cr}$ could be fitted with $\alpha=1.3$, which reduced to $\alpha=1$ after annealing. 
(a) Before anneal
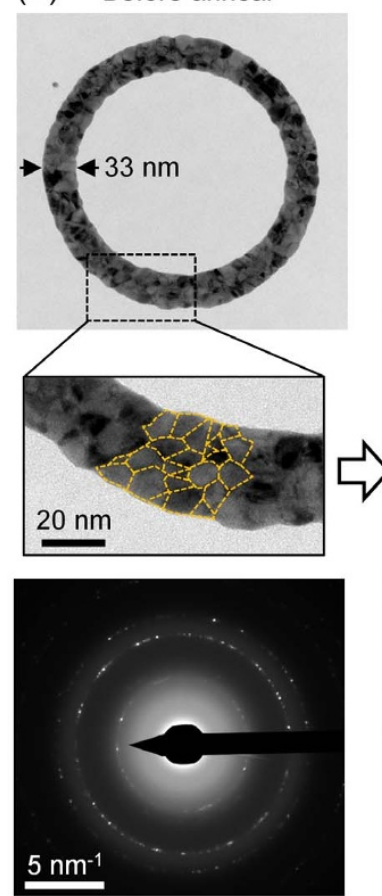

(b)
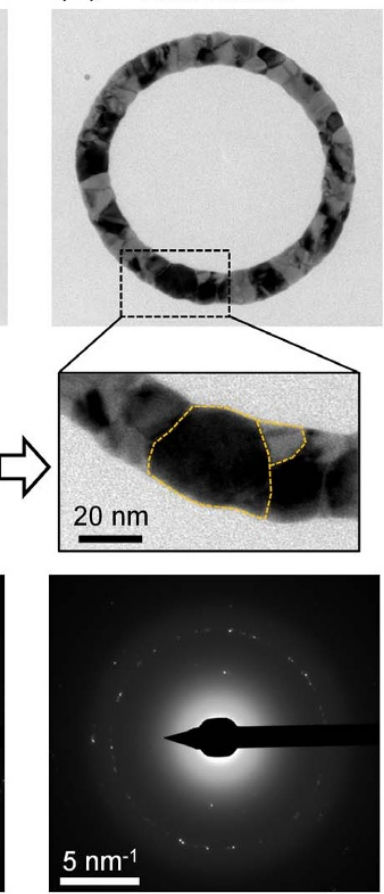

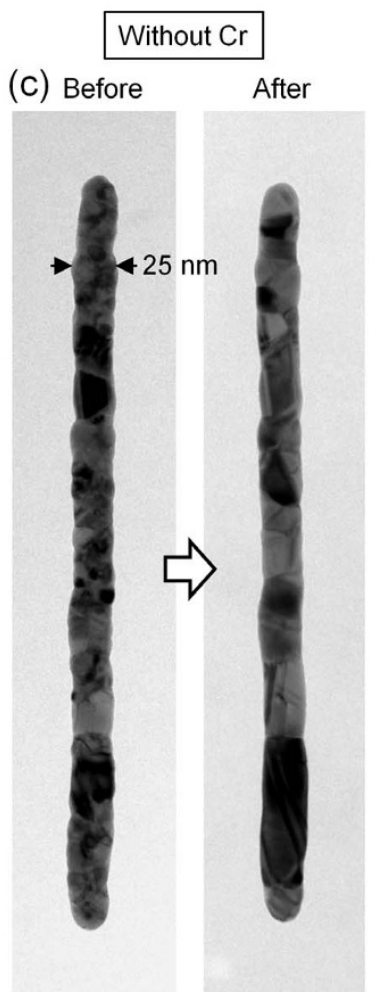

Figure $2 \mid$ Reducing the density of grain boundaries with shape preservation of EBL-defined nanostructures. Panels (a, b) show structures with Cr adhesion layers, while the rods in panel (c) have no adhesion layer. TEM image and electron diffraction pattern of the same ring structure before (a) and after annealing (b), showing a reduction in the density of grains and grain boundaries. (c) TEM images of a rod structure before and after annealing.

\section{Discussion}

Annealing strongly enhances the Q-factors for plasmons with resonance energies below $\sim 1.7 \mathrm{eV}$. This effect can be understood from the relatively low-frequency Drude damping parameter in $\mathrm{Au}, \Gamma_{p} \sim$ $0.1 \mathrm{eV}$. Therefore, only resonances that occur at low frequencies are most strongly affected by a modification to this parameter. Consistently, Fig. S2 in the SI illustrates that the damping factor affects only the imaginary part of the permittivity at energies below $\sim 1.7 \mathrm{eV}$. The decrease in Q-factors $>1.7 \mathrm{eV}$ is due to damping from interband transitions in $\mathrm{Au}^{4}$.

Figure 4(a) shows that the wet-chemically synthesized nanorods have comparable but systematically higher Q-factors than the annealed, EBL-patterned nanostructures. We identified two main reasons for this observation: (1) smaller physical cross sections and volumes of the rounded single-crystal nanorods ${ }^{11,35,36}$, and (2) the smaller contact area of these nanorods with the substrate. The widths of the measured wet-chemically synthesized nanorods were in the range of 17 to $32 \mathrm{~nm}$ while the EBL patterned nanostructures were systematically wider, ranging from 23 to $32 \mathrm{~nm}$. To enhance mutual comparison, the simulations used $25 \mathrm{~nm}$ as the lateral widths for all the simulated structures. As shown in Fig. 4(a), numerical simulations using $\alpha=1$ for structures with a pentagonal cross section (blue line) agree well with experimental data for the single-crystal nanorods. In reality, the cross sections of our wet-chemically synthesized particles are neither pentagonal nor perfectly circular $^{37}$, but somewhere in-between, see for example Fig. S4 in the SI. As a result, their Q-factors would fall between the blue and black solid lines in Fig. 4(b), the latter of which simulates nanostructures with rounded corners.

The normalized E-field magnitude distributions of gold nanostructures with two different lengths for points I-IV are simulated in the panels on the right of Fig. 4. For resonances around $1 \mathrm{eV}$ (panels I, II), the field distributions are comparable for both the square and pentagonal cross sections. At these low energies, radiative damping dominates. As a consequence, their relatively smaller volume leads to a somewhat higher Q-factor for pentagonal nanorods $^{32}$. This effect is confirmed in Fig. 4(b), where at low energies, structures with smaller cross-sectional areas (i.e. smaller volumes) have higher Q-factors. Conversely, for high-energy resonances around $2.1 \mathrm{eV}$ (panels III, IV), absorptive damping dominates and the stronger field localization at the metal-substrate interface dictates that structures with the smallest contact areas have the highest Q-factors, as seen in Fig. 4(b).

The effect of the substrate is further studied by comparing Qfactors for structures with and without substrates, also plotted in Fig. 4(b). For a given cross section, the presence of a substrate improves the Q-factor at low energies, but strongly reduces the Qfactor at high energies. The different effects of the substrate arise again from the interplay between radiative and absorptive damping. The presence of a substrate reduces radiative damping at low energies, by localizing fields at the metal-substrate interface, and leads to an overall increase in Q-factor, see also Figures S5 and S6 in the SI. On the other hand, this substrate effect leads to a decrease in Q-factor at high energies due to the enhanced absorptive damping at the metal-substrate interface. The sensitive dependence on the metalsubstrate contact area can also explain the scatter in the data of Fig. 4(a) for single-crystal nanorods: Some of these rods are likely to contact the substrate only at localized points, unlike the conformal contact in lithographically-defined structures.

It is worth pointing out that even though grain boundaries remain after annealing, the damping factors of the annealed structures still match those of single-crystal nanorods. We predict that the effect still of grain boundaries on damping strongly depends on their orientation, their position along the particle length with respect to the plasmon resonance nodes, and whether low- or high-angle grain boundaries are present ${ }^{38}$. The crystallographic defect density can vary greatly between different types of grain boundaries. For an isotropic material such as gold, defect-free twin boundaries 

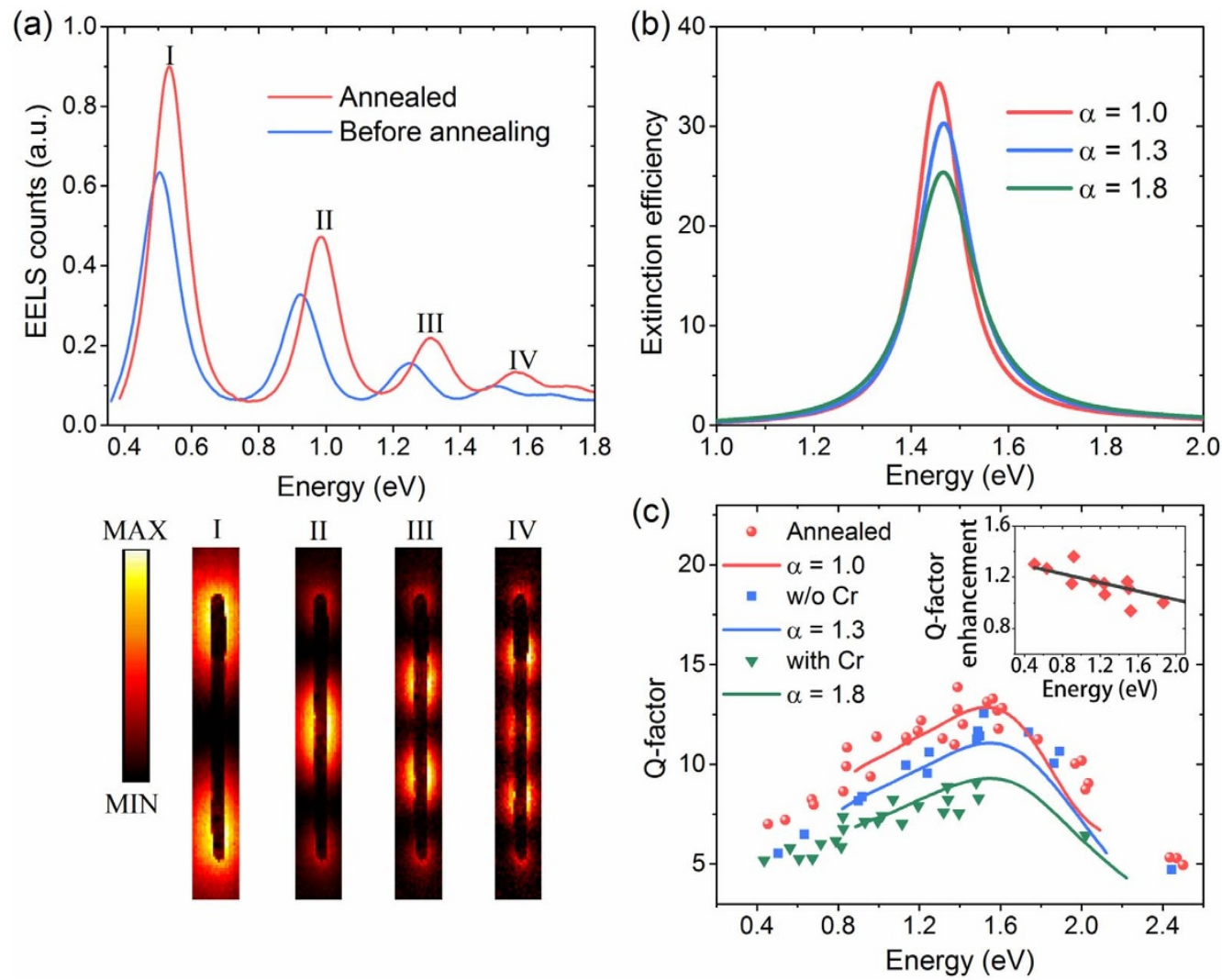

Figure $3 \mid$ Effect of encapsulated annealing and the adhesion layer on plasmon damping. (a) Experimental EELS spectra before and after annealing for the single nanostructure shown in Fig. 2c. The spectra were first normalized at the 'zero-loss peak' maximum, to allow mutual comparison of their intensities. Peaks I-IV with their corresponding EELS plasmon maps underneath show the first four harmonic resonance modes. (b) Simulated extinction efficiency showing peak broadening with increasing damping factor $\alpha$. (c) Q-factors measured from over 20 nanostructures from samples fabricated with a Cr adhesion layer (green triangles), without any adhesion layer before annealing (blue squares), and after encapsulated annealing (red disks). Solid lines are numerical simulations with $\alpha$ chosen to achieve good agreement with experimental data. Inset: Q-factor enhancement as a function of resonance energy.

potentially introduce less damping than high-angle grain boundaries (having grain misorientations $>10^{\circ}$ ) loaded with crystallographic defects that act as scattering centers for electrons ${ }^{31}$. We therefore hypothesize that the large plasmon damping in as-deposited samples is predominantly due to the presence of high-angle grain boundaries. As grain-boundary mobility increases with the degree of misorientation, these high-angle grain boundaries are also most easily removed by annealing. Our results suggest that the boundaries that remain are predominantly low-angled ones that have little effect on plasmon damping. The annealed structures, while polycrystalline, are therefore optically indistinguishable from a single crystal. Finally, over the entire energy range that was investigated, surface roughness of the nanostructures was found to have very little effect on the Q-factor ${ }^{4,7}$, as can be seen in Fig. S7 in the SI.

In answer to our introductory question, we demonstrated that lithographically-defined nanostructures experience more plasmon damping than wet-chemically synthesized metal nanoparticles due to the combined effect of grain boundaries, the larger metal-substrate interface, and metal adhesion layers. We presented a facile process to improve the Q-factors of nanostructures that are fabricated using top-down lithography. Encapsulation by HSQ, followed by annealing and HSQ removal resulted in polycrystalline gold nanostructures with Q-factors comparable to those of single-crystal nanorods. Both structures could be modelled using damping parameters of bulk gold. Our results provide design guidelines that indicate the dominant source(s) of damping for a given energy range, and will enable the fabrication of high Q-factor plasmonic resonators and antennas. If left intact, the HSQ encapsulation could also prevent degradation of metal resonators operating at elevated temperatures, such as in near-field transducers for heat-assisted magnetic recording, and photoelectron-emission cathodes.

\section{Methods}

EELS experiments were performed in scanning TEM (STEM) mode using an FEI Titan TEM with a Schottky electron source. The microscope was operated at $80 \mathrm{kV}$, using a STEM probe with a diameter around $1 \mathrm{~nm}$. With a Wien-type monochromator, an energy resolution with typical FWHM values of $70 \mathrm{meV}$ was obtained. Mapping and spectroscopy was done with a Gatan Tridiem ER EELS detector, applying a $7 \mathrm{mrad}$ collection semiangle using STEM-based spectrum imaging and a modified binned gain averaging acquisition routine ${ }^{39}$. The EELS spectra in Fig. 3 were taken by summing all EELS spectra from the spectrum image in the first $5 \mathrm{~nm}$ off the surface of the whole nanostructure. The resulting summed EELS spectra are therefore representative of the nanostructure as a whole. The spectra were normalized at the 'zero-loss peak' maximum, making it possible to mutually compare the spectral intensity before and after annealing. The background signal was measured from a bare amorphous $\mathrm{Si}_{3} \mathrm{~N}_{4}$ TEM support membrane, fitted to- and subtracted from the experimental EELS plasmon spectra. EELS maps show the loss signal integrated over an energy window of $0.1 \mathrm{eV}$ energy around the plasmon peaks of interest. Q-factors were calculated from the first and-when possible-higher-order plasmon modes ${ }^{40}$ in the EELS spectra, as described in detail elsewhere ${ }^{32}$, by applying Olivero and Longbothum's accurate empirical approach ${ }^{41}$. Q-factors were extracted from the most prominent peaks for a given nanostructure, up to the third-order harmonic.

Synthesis of gold nanorods. Gold(III) chloride trihydrate $\left(\mathrm{HAuCl}_{4} \cdot 3 \mathrm{H}_{2} \mathrm{O}, \geq 99.9 \%\right.$ trace metals basis), Sodium citrate dihydrate ( $\geq 99 \%)$, hexadecyltrimethylammonium bromide (CTAB, $\geq 99 \%$, powder), and L-ascorbic acid (Biotra, $\geq 99.0 \%$ ) were purchased from Sigma-Aldrich Singapore Pte Ltd. Ultra-pure deionized water (ELGA pure lab water systems) was used to prepare all aqueous solutions. The preparation of seeds $\sim 3.5 \mathrm{~nm}$ in diameter was done following the three-step seedmediated method introduced by Jana et $\mathrm{l}^{42}$. A $20 \mathrm{ml}$ of gold seeds aqueous solution containing $\mathrm{HAuCl}_{4} \cdot 3 \mathrm{H}_{2} \mathrm{O}\left(2.5 \times 10^{-4} \mathrm{M}\right)$ and tri-sodium citrate $\left(2.5 \times 10^{-4} \mathrm{M}\right)$ was 

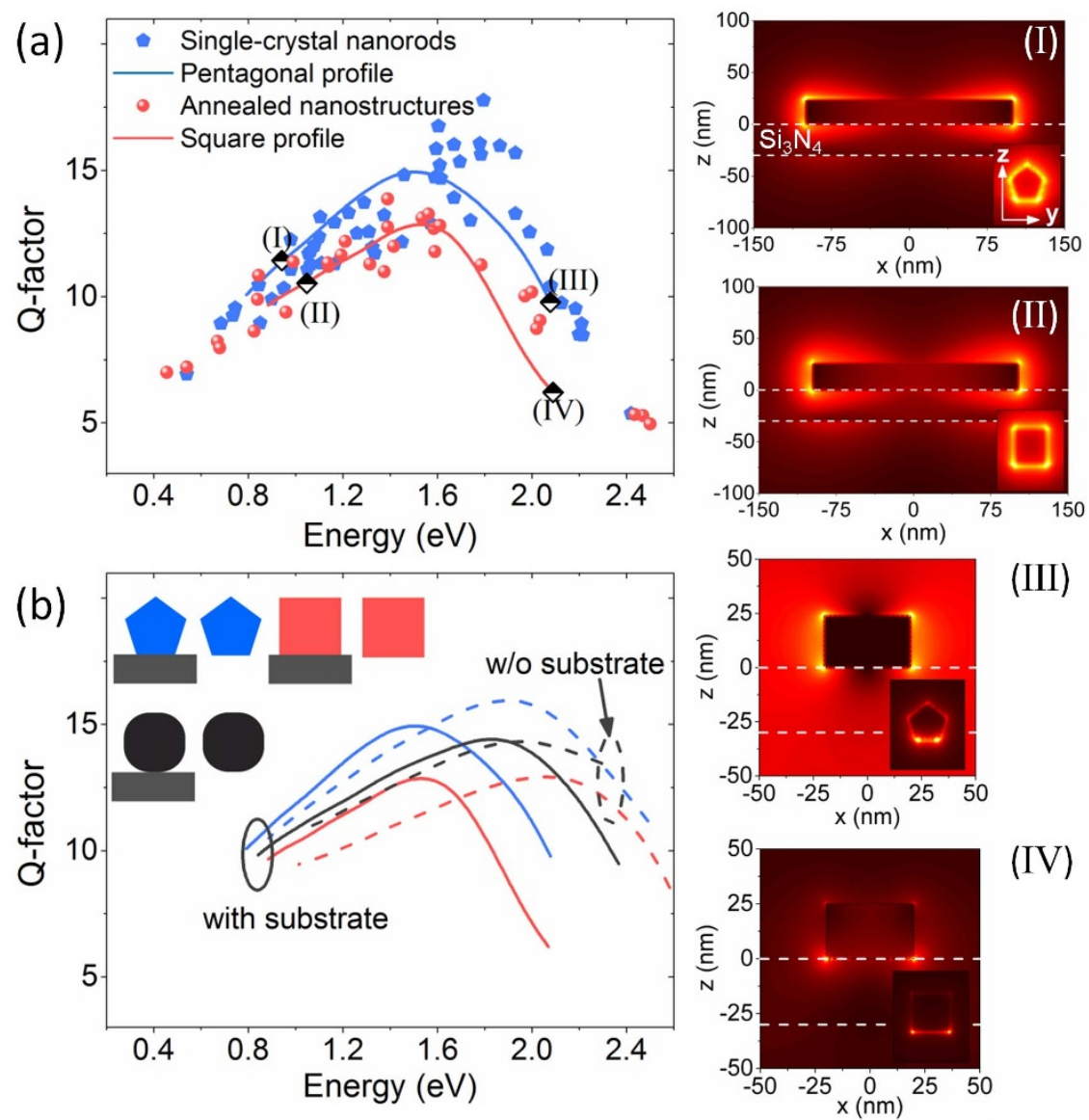

Figure $4 \mid$ Effect of the substrate and the interface on plasmon damping. (a) Experimental Q-factor plot for annealed, EBL-defined structures (red disks), and chemically synthesized, single-crystal nanorods (blue pentagons). The right-hand panels show the $|\mathrm{E}|$ field distributions, normalized at their maxima (side view and edge-on views (inset)) for points I-IV. (b) Simulated Q-factors for structures with a square cross section (red lines), a pentagonal cross section (blue line), and a rounded cross section (black line), all using $\alpha=1$. Dashed lines are simulated Q-factors in the absence of the $\mathrm{Si}_{3} \mathrm{~N}_{4}$ substrate.

prepared in a $22 \mathrm{ml}$ glass vial. Then, $0.6 \mathrm{ml}$ of an aqueous ice-cold $\mathrm{NaBH}_{4}(0.1 \mathrm{M})$ was added at once into the growth solution. The solution was allowed to stir for 2 hours before proceeding to the next step. A $30 \mathrm{ml}$ of growth solution containing $\mathrm{CTAB}(0.1 \mathrm{M})$ and $\mathrm{HAuCl}_{4} \cdot 3 \mathrm{H}_{2} \mathrm{O}\left(2.5 \times 10^{-4} \mathrm{M}\right)$ was prepared. This growth solution was distributed into three polypropylene tubes (labeled A, B and C) with $9 \mathrm{ml}$ each. Next, $0.05 \mathrm{ml}$ of ascorbic acid $(0.1 \mathrm{M})$ was added into each tube followed by gentle inversion mixing. Then, $1.0 \mathrm{ml}$ of the gold seeds solution was added into tube A. After $15 \mathrm{~s}, 1.0 \mathrm{ml}$ from tube A was transferred into tube B. After another $15 \mathrm{~s}$, $1.0 \mathrm{ml}$ from tube $\mathrm{B}$ was transferred from tube $\mathrm{B}$ into tube $\mathrm{C}$. Solution in tube $\mathrm{C}$ which contained gold nanorods, spheres and plates, was subjected to centrifugation for shape separation. Typically, $1.5 \mathrm{ml}$ of the particle solution was centrifuged at $2000 \mathrm{rpm}$ for $6 \mathrm{~min}$ repeatedly. The supernatant was poured off and the solid part containing nanorods and plates was re-dispersed in about $0.1 \mathrm{ml}$ of deionized water, from which a droplet was dried on a $\mathrm{Si}_{3} \mathrm{~N}_{4}$ TEM support membrane.

Simulations. The commercial finite-difference time-domain (FDTD) code from LUMERICAL was used to simulate the damping parameters and field distributions. A plane wave with the electric field polarized along the long axis of the nanorod illuminates a nanorod at normal incident angle. Perfectly matched layer (PML) boundary conditions encompass one isolated nanorod. For samples fabricated by EBL, a square cross section, $25 \mathrm{~nm}$ wide, was set for all the relevant simulations regardless of annealing condition or adhesion layer, while for the wet-chemically synthesized particles, a $25 \mathrm{~nm}$ wide pentagonal cross section was applied. Electric field plots were normalized to their maxima. Q-factors were obtained by fitting the fundamental mode of the extinction spectra with a Lorentzian function, using nanorods with lengths varying from 40 to $275 \mathrm{~nm}$. The relative permittivity for $\mathrm{Si}_{3} \mathrm{~N}_{4}$ was set to be 4 .

1. Im, H., Bantz, K. C., Lindquist, N. C., Haynes, C. L. \& Oh, S.-H. Vertically oriented sub-10-nm plasmonic nanogap arrays. Nano Lett. 10, 2231-2236 (2010).

2. Duan, H., Hu, H., Kumar, K., Shen, Z. \& Yang, J. K. W. Direct and reliable patterning of plasmonic nanostructures with sub-10-nm gaps. ACS Nano $\mathbf{5}$, 7593-7600 (2011).
3. Duan, H., Hu, H., Hui, H. K., Shen, Z. \& Yang, J. K. W. Free-standing sub-10 nm nanostencils for the definition of gaps in plasmonic antennas. Nanotechnology $\mathbf{2 4}$, 185301 (2013).

4. Sönnichsen, C. et al. Drastic reduction of plasmon damping in gold nanorods. Phys. Rev. Lett. 88, 077402 (2002).

5. Zhang, L., Chan, C. Y., Li, J. \& Ong, H. C. Rational design of high performance surface plasmon resonance sensors based on two-dimensional metallic hole arrays. Opt. Express 20, 12610-12621 (2012).

6. Nagpal, P., Lindquist, N. C., Oh, S.-H. \& Norris, D. J. Ultrasmooth patterned metals for plasmonics and metamaterials. Science 325, 594-597 (2009).

7. Chen, K.-P., Drachev, V. P., Borneman, J. D., Kildishev, A. V. \& Shalaev, V. M. Drude relaxation rate in grained gold nanoantennas. Nano Lett. 10, 916-922 (2010).

8. Sambles, J. R. Grain-boundary scattering and surface plasmon attenuation in noble metal films. Solid State Commun. 49, 343-345 (1984).

9. Habteyes, T. G. et al. Metallic adhesion layer induced plasmon damping and molecular linker as a nondamping alternative. ACS Nano 6, 5702-5709 (2012).

10. Liu, Z. et al. Plasmonic nanoantenna arrays for the visible. Metamaterials 2, 45-51 (2008).

11. Critchley, K. et al. Near-bulk conductivity of gold nanowires as nanoscale interconnects and the role of atomically smooth interface. Adv. Mater. 22, 2338-2342 (2010).

12. Huang, J.-S. et al. Atomically flat single-crystalline gold nanostructures for plasmonic nanocircuitry. Nat. Commun. 1, 150 (2010)

13. Jung, Y. S., Sun, Z., Kim, H. K. \& Blachere, J. Blueshift of surface plasmon resonance spectra in anneal-treated silver nanoslit arrays. Appl. Phys. Lett. 87, 263116 (2005).

14. Tinguely, J.-C. et al. Gold nanoparticles for plasmonic biosensing: the role of metal crystallinity and nanoscale roughness. BioNanoSci. 1, 128-135 (2011).

15. Liu, H. et al. Enhanced surface plasmon resonance on a smooth silver film with a seed growth layer. ACS Nano 4, 3139-3146 (2010).

16. Karakouz, T. et al. Mechanism of morphology transformation during annealing of nanostructured gold films on glass. Phys. Chem. Chem. Phys. 15, 4656-4665 (2013). 
17. Karim, S. et al. Influence of crystallinity on the Rayleigh instability of gold nanowires. J. Phys. D Appl. Phys. 40, 3767 (2007).

18. Nie, S. \& Emory, S. R. Probing single molecules and single nanoparticles by surface-enhanced Raman scattering. Science 275, 1102-1106 (1997).

19. Kumar, K. et al. Printing colour at the optical diffraction limit. Nat. Nanotechnol. 7, 557-561 (2012)

20. Curto, A. G. et al. Unidirectional emission of a quantum dot coupled to a nanoantenna. Science 329, 930-933 (2010).

21. Yang, J. K. W., Bosman, M., Duan, H., Kumar, K. \& Phang, I. Y. Process for creating lithographically-defined plasmonic structures with enhanced Q-factors. US Patent Application no. US 20130243947 A1. (2013).

22. Liu, N. et al. Plasmonic analogue of electromagnetically induced transparency at the Drude damping limit. Nat. Mater. 8, 758-762 (2009).

23. Bosman, M., Keast, V. J., Watanabe, M., Maaroof, A. I. \& Cortie, M. B. Mapping surface plasmons at the nanometre scale with an electron beam. Nanotechnology 18, 165505 (2007)

24. Nelayah, J. et al. Direct imaging of surface plasmon resonances on single triangular silver nanoprisms at optical wavelength using low-loss EFTEM imaging. Opt. Lett. 34, 1003-1005 (2009).

25. Rossouw, D., Couillard, M., Vickery, J., Kumacheva, E. \& Botton, G. A. Multipolar plasmonic resonances in silver nanowire antennas imaged with a subnanometer electron probe. Nano Lett. 11, 1499-1504 (2011).

26. Duan, H., Fernández-Domínguez, A. I., Bosman, M., Maier, S. A. \& Yang, J. K. W. Nanoplasmonics: classical down to the nanometer scale. Nano Lett. 12, 1683-1689 (2012).

27. Kociak, M. \& Stéphan, O. Mapping plasmons at the nanometer scale in an electron microscope. Chem. Soc. Rev. 43, 3865-3883 (2014).

28. Tan, S. F. et al. Quantum plasmon resonances controlled by molecular tunnel junctions. Science 343, 1496-1499 (2014)

29. Koh, A. L., McComb, D. W., Maier, S. A., Low, H. Y. \& Yang, J. K. W. Sub-10 nm patterning of gold nanostructures on silicon-nitride membranes for plasmon mapping with electron energy-loss spectroscopy. J. Vac. Sci. Technol. B 28 C6O45-C46O49 (2010).

30. Koh, A. L., Fernández-Dominguez, A. I., McComb, D. W., Maier, S. A. \& Yang, J. K. W. High-resolution mapping of electron-beam-excited plasmon modes in lithographically defined gold nanostructures. Nano Lett. 11, 1323-1330 (2011).

31. Humphreys, F. J. \& Hatherly, M. Recrystallization and related annealing phenomena. (Pergamon, 2004)

32. Bosman, M. et al. Surface plasmon damping quantified with an electron nanoprobe. Sci. Rep. 3, 1312 (2013).

33. Palik, E. D. Handbook of optical constants, Vol. III. (Academic Press, San Diego; 1998).

34. Moody, N. R. et al. Effects of diffusion on interfacial fracture of gold-chromium hybrid microcircuit films. Int. J.Fracture 120, 407-419 (2003).

35. Johnson, C. J., Dujardin, E., Davis, S. A., Murphy, C. J. \& Mann, S. Growth and form of gold nanorods prepared by seed-mediated, surfactant-directed synthesis. J. Mater. Chem. 12, 1765-1770 (2002).

36. Hu, H. et al. Photoluminescence via gap plasmons between single silver nanowires and a thin gold film. Nanoscale 5, 12086-12091 (2013).

37. Katz-Boon, H. et al. Three-dimensional morphology and crystallography of gold nanorods. Nano Lett. 11, 273-278 (2011)
38. Bietsch, A. \& Michel, B. Size and grain-boundary effects of a gold nanowire measured by conducting atomic force microscopy. Appl. Phys. Lett. 80, 3346-3348 (2002).

39. Bosman, M. \& Keast, V. J. Optimizing EELS acquisition. Ultramicroscopy 108, 837-846 (2008).

40. Schmidt, F.-P. et al. Universal dispersion of surface plasmons in flat nanostructures. Nat. Commun. 5, 3604 (2014).

41. Olivero, J. J. \& Longbothum, R. L. Empirical fits to the Voigt line width: A brief review. J. Quant. Spectrosc. Radiat. Transfer 17, 233-236 (1977).

42. Jana, N. R., Gearheart, L. \& Murphy, C. J. Wet chemical synthesis of high aspect ratio cylindrical gold nanorods. J. Phys.Chem. B 105, 4065-4067 (2001).

\section{Acknowledgments}

This work was supported by the Agency for Science, Technology and Research (A*STAR) in Singapore and made use of the SnFPC facilities in IMRE. J.K.W.Y. kindly acknowledges support from the Singapore National Research Foundation (NRF) under the CRP program (Award Nos. NRF-2011, NRF-CRP 001-126) and the NRF is acknowledged by J.K.W.Y., M.B. and C.A.N. for support via NRF-CRP 8-2011-07). L.Z. and C.-W.Q. acknowledge financial support from the National University of Singapore (Grant No R-263-000-688112). H.D. acknowledges financial support from the National Science Foundation of China (Grant Nos 1274107 and 61204109).

\section{Author contributions}

J.K.W.Y. proposed the use of HSQ for encapsulated annealing, fabricated the TEM samples (together with H.D.), performed the annealing, and processed the EBL samples. M.B. performed TEM and EELS, and processed \& analyzed the EELS data. L.Z. and C.-W.Q. performed numerical simulations, S.F.T. and C.A.N. synthesized the gold nanorods. All authors contributed to the writing, analysis, and conclusions of the work.

\section{Additional information}

Supplementary information accompanies this paper at http://www.nature.com/ scientificreports

Competing financial interests: The authors declare no competing financial interests.

How to cite this article: Bosman, M. et al. Encapsulated Annealing: Enhancing the Plasmon Quality Factor in Lithographically-Defined Nanostructures. Sci. Rep. 4, 5537; DOI:10.1038 srep05537 (2014)

his work is licensed under a Creative Commons Attribution-NonCommercialNoDerivs 4.0 International License. The images or other third party material in this article are included in the article's Creative Commons license, unless indicated otherwise in the credit line; if the material is not included under the Creative Commons license, users will need to obtain permission from the license holder in order to reproduce the material. To view a copy of this license, visit http:// creativecommons.org/licenses/by-nc-nd/4.0/ 